\title{
Prevalência de depressão nos internos de medicina geral e familiar da região sul de Portugal Continental: um estudo multicêntrico
}

Ana Rita Machado Gomes*

\section{RESUMO}

Objetivos: Determinar a prevalência de depressão e consumo de antidepressivos nos internos de medicina geral e familiar (MGF) da região sul de Portugal Continental e estudar a associação da depressão com sexo, idade, ano de internato, localização do centro de saúde e tipo de unidade de cuidados de saúde.

Tipo de estudo: Observacional, transversal e descritivo.

Local: Unidades de Saúde Familiar (USF) e Unidades de Cuidados de Saúde Personalizados (UCSP) da região sul de Portugal Continental. População: Internos de medicina geral e familiar (MGF) da região sul do território português.

Métodos: Envio de questionários de autopreenchimento por via eletrónica aos internos de MGF da região sul de Portugal continental. O instrumento de medição de depressão usado foi o Inventário Depressivo de Beck II. Os testes Mann-Whitney, Kruskal-Wallis e Correlação de Spearman foram utilizados para avaliar associações de variáveis, considerando-se um nível de significância de 0,05 e intervalos de confiança a $95 \%$.

Resultados: A taxa de resposta foi de 33\%, reunindo-se uma amostra de 216 participantes. Quase $80 \%$ dos inquiridos pertencem ao sexo feminino. A média de idades foi 30 anos. Os internos distribuem-se equitativamente pelos quatro anos de formação pós-graduada. A maioria $(88,9 \%)$ desenvolve a sua atividade em USF e $61,1 \%$ trabalha na região de Lisboa. Cerca de $19 \%$ dos internos apresentam algum grau de depressão: $8,9 \%(4,7-13,0)$ com depressão leve, 6,9\% $(3,2-10,6)$ com depressão moderada e 2,9\% $(0,4-5,5)$ com depressão severa. Sete por cento estão medicados com antidepressivos. Os valores na escala de depressão não se relacionam com idade, sexo, ano de internato e tipo de unidade.

Conclusões: O estudo representa o primeiro esforço de quantificação de depressão nos internos de MGF em Portugal e poderá configurar um ponto de partida para outros estudos no âmbito da saúde mental dos médicos que resultem em intervenções que tendam a melhorar a prática clínica dos mesmos.

Palavras-chave: Internato; Medicina Familiar; Depressão.

\section{INTRODUÇÃO}

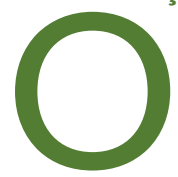

internato médico é atualmente reconhecido como um período de risco para o desenvolvimento de vários problemas de saúde mental, incluindo a depressão. ${ }^{1-12} \mathrm{Na}$ literatura, este assunto cruza-se frequentemente com conceitos complexos e difíceis de padronizar, como burnout, qualidade de vida, perceção de stress, bem-estar psicológico, qualidade/quantidade de sono, satisfação profissional/carreira, performance profissional e autoperceção do erro. Na sua maioria, os estudos

*Médica de Medicina Geral e Familiar. USF Carcavelos - ACES Cascais publicados são transversais, limitados a populações de internos hospitalares, que reportam uma prevalência de depressão maior nos internos relativamente à população geral.

No âmbito estrito do internato de medicina familiar, a informação é mais escassa e os resultados contraditórios quanto à prevalência de depressão nesta população relativamente à população geral. ${ }^{5-12}$

No contexto nacional encontram-se publicados artigos apenas sobre o burnout médico. Nenhum com foco no problema da depressão no médico interno e sua quantificação.

A depressão no interno de medicina geral e familiar (MGF) pode ser equacionada numa lógica de produtividade e qua- 
lidade clínica e académica, por um lado, e na dimensão puramente humana, por outro. Do primeiro tópico destaca-se, de imediato, a associação, provavelmente bidirecional, entre a depressão e o erro médico. ${ }^{4} \mathrm{O}$ segundo ponto remete para a dimensão extra profissional do interno como indivíduo em risco, ou seja, como indivíduo capaz de adoecer por razões múltiplas, incluindo aquelas intrínsecas à sua atividade formativa e profissional que o expõem a stressors repetidamente assinalados na literatura. ${ }^{13-14}$

Os objetivos deste estudo são determinar a prevalência de depressão e consumo de antidepressivos nos internos de MGF da região sul do território português e estudar a associação da depressão com as variáveis: sexo, idade, ano de internato, localização do centro de saúde e tipo de unidade de cuidados de saúde.

\section{MÉTODOS}

Estudo observacional, transversal e descritivo, baseado num questionário eletrónico, cujo desenho metodológico foi orientado pelas normas CHERRIES, para desenvolver o protocolo de recolha de dados utilizando a internet, e STROBE, para reportar os resultados.

O protocolo foi previamente submetido e aprovado pela Comissão de Ética da Administração Regional de Saúde de Lisboa e Vale do Tejo.

O procedimento consistiu no envio de um email-convite, com o link para um questionário eletrónico, contínuo, página única, de autopreenchimento voluntário, criado através da aplicação Google Drive®, a todos os internos de MGF da região sul (Lisboa e Vale do Tejo, Alentejo e Algarve). O envio dos questionários foi realizado a 22 de julho de 2014 pela Comissão de Internos de MGF da região sul, que utilizou a sua base de dados de contactos eletrónicos. O consentimento para entrar no estudo foi considerado automático caso o interno respondesse ao questionário. Este ponto encontrava-se explicado no texto informativo do email-convite.

Foram aceites as respostas enviadas entre 22 de julho e 12 de setembro de 2014, período oficial de recolha de dados. Durante este período, o email-convite foi reenviado duas vezes, para criar oportunidade renovada de divulgação e apelo à participação. No mesmo período, a página oficial da Coordenação do Internato da MGF da Lisboa eVale do Tejo anunciou o estudo.

O questionário apresentava sete questões de escolha múltipla pré-estabelecida. As cinco primeiras correspondiam às variáveis independentes - sexo, idade, ano de internato, localização do centro de saúde e tipo de unidade de cuidados de saúde. As duas últimas diziam respeito às variáveis dependentes em estudo - depressão, medida através do Inven- tário Depressivo de Beck II (IDB-II), e consumo atual de antidepressivos, definido como «consumo de forma diária, desde há pelo menos 14 dias, de um ou mais fármacos da família dos antidepressivos».

O IDB-II é uma escala não-diagnóstica de autoavaliação de depressão, constituído por 21 dimensões de sintomas e atitudes. Cada dimensão é constituída por quatro frases, com valores de 0 a 3 pontos, dispostas por ordem crescente de intensidade. O indivíduo seleciona a frase que melhor define $o$ estado emocional vivenciado nas duas últimas semanas, incluindo o dia do questionário. A pontuação total do questionário situa-se entre os 0 e os 63 pontos, consoante as respostas dos indivíduos. Onze dimensões do questionário relacionam-se com aspetos cognitivos, cinco com comportamentos observáveis, duas com o afeto e uma com sintomas interpessoais. Após soma das cotações, o resultado pode ser expresso numa das categorias de depressão definidas: 0-13, depressão mínima; 14-19, depressão leve; 20-28, depressão moderada; 29-63, depressão severa. Assim, considera-se existir algum grau de depressão a partir dos 14 pontos e ausência de depressão no primeiro nível da escala (score entre 0 e 13), apesar da designação «depressão mínima». O IDB-II foi aferido por Campos e Gonçalves para a população portuguesa, demonstrando estrutura muito semelhante à versão original. ${ }^{15}$

O resultado individual da escala de avaliação de sintomas foi enviado a todos os internos que o solicitaram, respondendo «sim» à pergunta criada para o efeito. O retorno do resultado individual no IDB-II foi o único incentivo pré-determinado para responder ao questionário.

O questionário apresentava ainda um campo para identificação através do número da ordem dos médicos, necessário para evitar repetições de respostas tendo em conta as limitações da aplicação informática utilizada. Posteriormente, de modo a preservar o anonimato e assegurar a confidencialidade, o colaborador externo codificou as respostas, considerando apenas a última no caso de repetições. Os questionários originais permaneceram arquivados em formato digital, com o colaborador externo, até ter sido terminado o processamento dos dados e realizado o retorno do resultado do IDB-II aos participantes interessados. Após este período, os ficheiros foram eliminados.

Foi realizada análise descritiva, com componente analítica, utilizando os softwares SPSS®, versão 22 e o Epidat ${ }^{\circledR}$, sendo a última ferramenta utilizada exclusivamente para o cálculo dos intervalos de confiança. Incluíram-se questionários com resposta incompleta, facto que implicou tratamento estatístico, considerando dados em falta com avaliação das proporções válidas. A normalidade das variáveis foi pesquisada 
previamente à escolha do teste estatístico. A correlação de Pearson foi aplicada para comparação no caso de variáveis independentes numéricas normais e a correlação de Spearman foi aplicada para comparação no caso de variáveis independentes numéricas não normais, os testes $t$-Student $\mathrm{e}$ Mann-Whitney para variáveis categoriais com duas categorias, respetivamente normais e não normais, e os testes Anova one-way e Kruskal-Wallis para variáveis categoriais com três ou mais categorias, respetivamente normais e não normais. O nível de significância considerado foi de 0,05 , com intervalos de confiança a 95\%.

\section{RESULTADOS}

A Comissão de Internos de MGF da região sul enviou um total de 655 questionários por via eletrónica. No período de 22 de julho a 12 de setembro de 2014 foram rececionadas 216 respostas válidas não repetidas. A taxa de resposta foi de $33 \%$. Assinale-se que as 216 respostas consideradas válidas incluíram 13 questionários sem número de identificação, cuja análise comparativa com as restantes respostas permitiu concluir não se tratarem de questionários repetidos.

Relativamente à caracterização das variáveis independentes, quanto ao sexo, dos 216 internos respondedores, 172 (80\%) pertenciam ao sexo feminino (Quadro I). A média das idades foi de 30 anos, a mediana 29 e a moda 28, com um mínimo de 25 e máximo de 57. Os internos estavam distribuídos de forma equitativa pelos quatro anos de formação pósgraduada. O mesmo não acontecia relativamente à distribuição regional. Mais de metade da amostra, 132 (62,6\%) dos internos, trabalhava em centros de saúde da região de Lisboa. A segunda região mais representada foi Setúbal, com 39 (18,5\%). As regiões de Portalegre, Baixo Alentejo e Alto Alentejo apenas estavam representadas com um, três e quatro internos na amostra, respetivamente. Quanto ao tipo de unidade onde desenvolviam a sua atividade clínica, 192 (89,3\%) internos encontravam-se colocados em USF.

No subgrupo específico dos internos de Lisboa e Vale do Tejo (Lisboa, Ribatejo e Setúbal), cuja coordenação divulgou informação relativa à proporção feminino/masculino da população de internos no período de recolha de dados, procedeu-se à comparação desta proporção com a da amostra. Assim, neste subgrupo, o teste binomial indica que a proporção de indivíduos do sexo masculino na amostra (18,2\%) foi inferior à da população (26,4\%), p=0,005.

Na amostra do estudo, excluindo o primeiro nível da escala (0 e 13), 18,7\% dos internos apresenta algum grau de depressão, ou seja, pontuação maior ou igual a 14 no Inventário Depressivo de Beck II. A média de pontuação nesta escala foi de 8,64, com um mínimo de 0 e um máximo de 50 pon- tos. A sua distribuição pelas categorias leve, moderada e severa do Inventário Depressivo foi, respetivamente, 8,9\% (4,713,0), 6,9\% (3,2-10,6) e 2,9\% (0,4-5,5) (Quadro II).

O Quadro III mostra as respostas dos internos em cada uma das 21 dimensões do Inventário. No topo do quadro, «Cansaço ou Fadiga» foi a dimensão com maior percentagem de respostas que se associam a alteração de intensidade variável. Seguiam-se «Perda de energia», «Alterações no Padrão de Sono» e «Dificuldade de Concentração». Em todas estas dimensões mais de metade dos internos assinalou resposta que se associa a alteração. A dimensão com menor frequência de respostas que representa alteração foi «Pensamentos ou Desejos Suicidas»; ainda assim, 4,2\% dos internos manifestou algum grau de alteração neste tópico.

Quanto ao consumo atual de antidepressivos, 7\% (3,4$10,7)$ dos internos respondedores afirmou estar a tomar este tipo de fármaco (Quadro II).

Nesta amostra, a depressão quantificada através do Inventário Depressivo de Beck II não se encontrava estatisticamente associada com as variáveis sexo, idade, ano de internato e tipo de unidade (Quadro IV). A hipótese de associação de depressão e localização do centro de saúde não foi testada, uma vez que esta última variável continha três categorias sem o número necessário de elementos para permitir a aplicação do teste estatístico.

O retorno do resultado da aplicação do Inventário Depressivo de Beck II foi realizado a 78\% ( $n=215)$ dos internos.

\section{DISCUSSÃO}

A amostra de internos apresentou uma prevalência de depressão de 18,7\%. Embora as limitações deste tipo de estudo impeçam a generalização e não esteja incluída a comparação com uma amostra de adultos jovens não médicos, este resultado vale, antes de mais, por ser a primeira tentativa de quantificação da depressão numa população de internos portugueses.

A prevalência encontrada não está afastada das apresentadas noutros estudos de prevalência de depressão em populações de internos de medicina familiar, nomeadamente o estudo transversal canadiano publicado em 2005, que serviu de referência principal ao presente trabalho e cujo objetivo era calcular a prevalência de depressão e ansiedade e descrever as estratégias de coping nos internos de medicina familiar da região de Ontário. Este trabalho apresentou uma prevalência total de depressão de $20 \%{ }^{7}$ Os seus autores utilizaram um modelo do Patient Health Questionnaire para medir depressão, também enviado na forma de inquérito online e obtiveram resposta a 254 questionários, com taxa de resposta de $37 \%{ }^{7}$ 
Na verdade, a literatura sobre prevalência de depressão nos internos mostra um intervalo amplo de percentagens. Ainda assim, a maioria dos autores, como os do estudo de referência relatado, concluem que a prevalência de depressão é significativamente superior nos internos quando se compara com a população geral da mesma região. Com grande probabilidade, parte significativa das diferenças nos resultados resultam da heterogeneidade entre os vários estudos: diferentes instrumentos de medida de depressão, diferentes subpopulações de internos e diferente metodologia. Em alguns casos são relatados valores de depressão superiores a $20 \%$. Num estudo americano, os autores relatam $25 \%$ de sintomas depressivos significativos em internos de várias especialidades que trabalham nos cuidados de saúde primários, incluindo medicina familiar, avaliados com 10-item Center for Epidemiologic Studies Depression Scale. ${ }^{6}$

Os estudos mais recentes, e com metodologia mais robusta, têm decorrido em populações de internos hospitalares. Em 2010, Sen etalapresentaram um estudo prospetivo que reuniu um coorte de 740 internos de 13 hospitais norte americanos de várias especialidades. Durante 14 meses e começando cerca de dois meses antes do início do internato, os participantes preencheram periodicamente um inquérito que incluía a escala 9-item Patient Health Questionnaire. Os resultados com significado estatístico revelaram um aumento marcado dos sintomas depressivos durante o internato médico:3,9\% dos internos com critérios de depressão no momento 0 versus $26,6 \%$ doze meses após início do internato. ${ }^{2}$

O 1. ${ }^{\circ}$ relatório do Estudo Epidemiológico Nacional de Saúde Mental ${ }^{16}$ apresenta a estimativa mais fiável de prevalência das perturbações do humor na população portuguesa - 7,9\%. Este valor é bastante inferior ao encontrado neste trabalho, apesar de incluir diferentes expressões patológicas das perturbações do humor. Contudo, as diferentes características das amostras em causa tornam precipitadas conclusões sobre um risco superior de depressão nos internos. De forma independente, de acordo com o $1 .^{\circ}$ relatório, o sexo feminino aumenta o risco de perturbação do humor (OR 2,3), bem como o facto de ter idade entre os 18 e 34 anos (OR2,18).
QUADRO I. Caracterização da amostra de participantes por sexo, idade, anos de internato, localização do centro de saúde e tipo de unidade de cuidados de saúde

\begin{tabular}{l|r|r|r} 
& $\mathbf{N}$ & \multicolumn{1}{|c|}{$\boldsymbol{n}(\%)$} & \multicolumn{1}{c}{$\mathbf{I C}_{95 \%}$} \\
\hline Sexo & 216 & & \\
Feminino & & $172(79,6)$ & $(74,03-85,23)$ \\
Masculino & & $44(20,4)$ & $(14,77-25,97)$ \\
\hline Idade & 213 & Média 30,19 & $(29,45-30,94)$ \\
\hline Ano de Internato & 216 & & \\
$1 .^{\circ}$ ano & & $50(23,1)$ & $(17,29-29,00)$ \\
$2^{\circ}$ ano & & $61(28,2)$ & $(22,00-34,48$ \\
3. $^{\circ}$ ano & & $49(22,7)$ & $(16,87-28,50)$ \\
$4 .^{\circ}$ ano & & $56(25,9)$ & $(19,85-32,00)$ \\
\hline Localização do centro de saúde & 211 & & \\
Algarve & & $16(7,6)$ & $(3,77-11,39)$ \\
Alto Alentejo & & $4(1,9)$ & $(0,52-4,78)$ \\
Baixo Alentejo & & $3(1,4)$ & $(0,29-4,10)$ \\
Lisboa & & $132(62,6)$ & $(55,79-69,32)$ \\
Portalegre & & $1(0,5)$ & $(0,01-2,61)$ \\
Santarém & & $16(7,6)$ & $(3,77-11,39)$ \\
Setúbal & & $39(18,5)$ & $(13,01-23,96)$ \\
\hline Tipo de unidade de cuidados de saúde & 215 & & \\
USF & & $192(89,3)$ & $(84,94-93,67)$ \\
UCSP & & $23(10,7)$ & $(6,33-15,06)$
\end{tabular}

\begin{tabular}{|c|c|c|c|}
\hline \multicolumn{4}{|c|}{$\begin{array}{l}\text { QUADRO II. Grau de depressão e consumo atual de } \\
\text { antidepressivos nos participantes do estudo }\end{array}$} \\
\hline & $\mathbf{N}$ & $n(\%)$ & $I_{95 \%}$ \\
\hline Depressão & 203 & & \\
\hline Depressão mínima [0-13] & & $165(81,3)$ & $(75,7-86,9)$ \\
\hline Depressão leve [14-19] & & $18(8,9)$ & $(4,7-13,0)$ \\
\hline Depressão moderada [20-28] & & $14(6,9)$ & $(3,2-10,6)$ \\
\hline Depressão grave [29-63] & & $6(2,9)$ & $(0,4-5,5)$ \\
\hline Consumo atual de antidepressivos & 213 & & \\
\hline «Sim» & & $15(7,0)$ & $(3,4-10,7)$ \\
\hline «Não» & & $198(93,0)$ & $(89,3-96,6)$ \\
\hline «Não sei» & & $0(0)$ & $(0-1,7)$ \\
\hline
\end{tabular}

A amostra do nosso estudo apresenta $80 \%$ de indivíduos do sexo feminino e uma média de idades de 30 anos, pelo que se esperariam valores superiores de «depressão». Por outro lado, mesmo que as características das amostras coincidissem, os dois trabalhos não estão a considerar exatamente o 


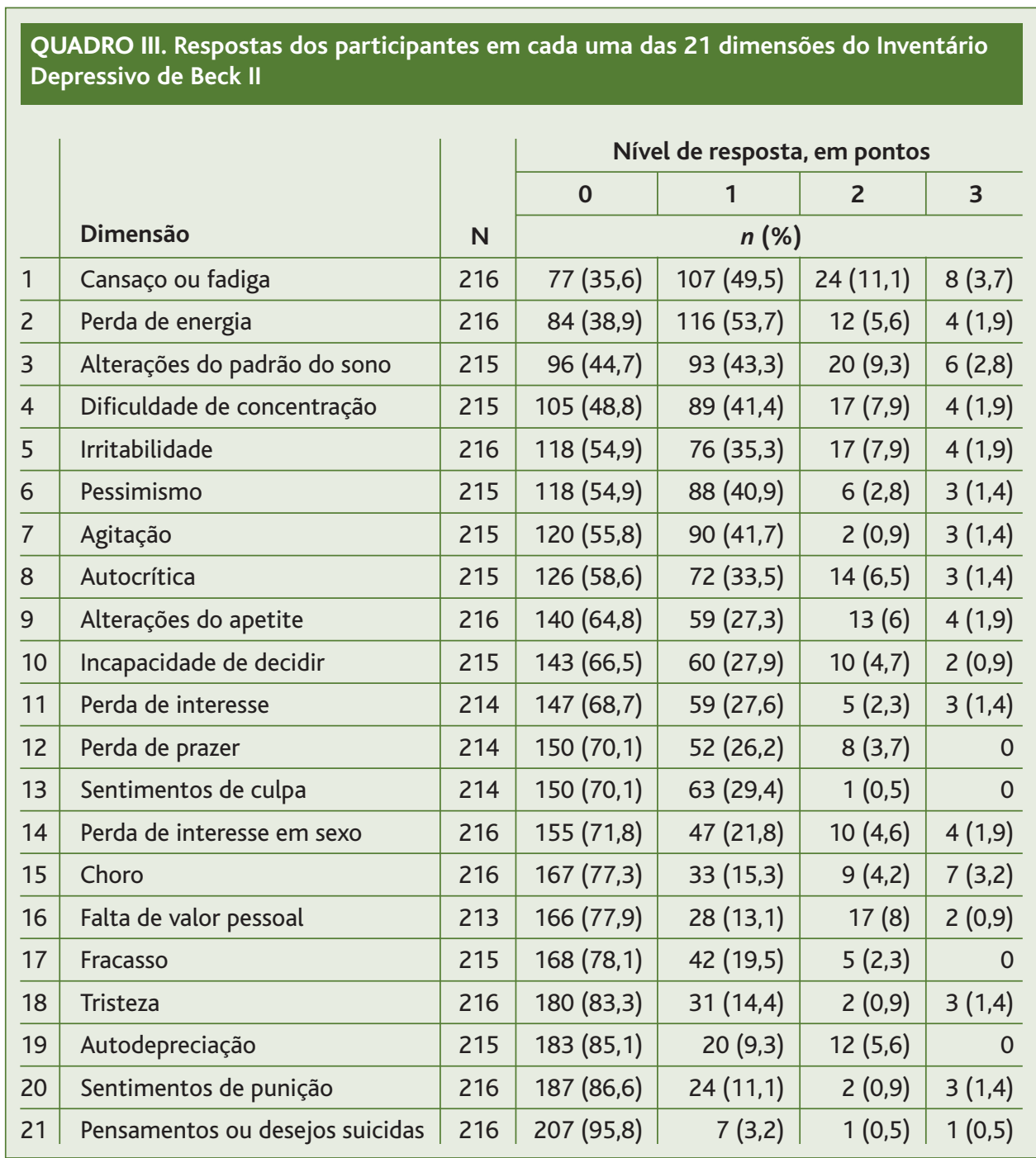

Nota - No Inventário Depressivo de Beck II cada dimensão é constituída por quatro frases (níveis de resposta), com valores de 0 a 3 pontos, dispostas por ordem crescente de intensidade. Na tabela, as 21 dimensões estão alinhadas da dimensão com maior percentagem de respostas diferentes de 0 para aquelas com menos. reta de saúde mental, ou seja, da gravidade da perturbação do humor. Contudo, o seu alcance pode ser equívoco. Por um lado, porque os antidepressivos não são utilizados apenas no tratamento de síndromas depressivos e, por outro, porque existem outras formas de tratamento ou coping de sintomas depressivos. No estudo canadiano, $13,4 \%$ dos internos usava medicação para lidar com a perturbação afetiva, 7,1\% realizava terapia cognitivacomportamental e 8,3\% interrompeu temporariamente a atividade clínica e formativa. ${ }^{7}$ Ao contrário do estudo canadiano, a análise das diferentes estratégias de $c o$ ping não está incluída nos objetivos deste trabalho.

Numa outra perspetiva, nenhuma das variáveis analisadas neste estudo apresentou associação com depressão. Relativamente ao sexo, também no estudo canadiano não se verificaram diferenças significativas. Todavia, na maioria dos trabalhos realizados neste contexto, o sexo feminino está associado a um risco aumentado de presença de sintomas de- mesmo problema e não o medem da mesma forma, pelo que não seria rigorosa uma comparação estatística direta. Apenas a comparação com resultados decorrentes da aplicação do Inventário Depressivo de Beck II numa amostra de nãomédicos internos de características equivalentes permitiria concluir com legitimidade sobre o aumento do risco de depressão na população de internos de MGF.

Na literatura são muito mais raros os estudos que quantificam o uso de medicação antidepressiva. Na nossa amostra, $7 \%$ dos internos afirmou estar a consumir antidepressivos. Neste âmbito, o parâmetro pretendia ser uma medida indi- pressivos. Ainda no estudo canadiano, verificou-se um incremento da prevalência de perturbações afetivas associada ao aumento dos anos de internato. ${ }^{7}$

Relativamente a limitações, salienta-se a taxa de resposta de $33 \%$ da população alvo do estudo. Na perspetiva teórica exclusiva não se pode considerar uma boa taxa de resposta. Contudo, dadas as limitações inerentes ao desenho do estudo, cuja metodologia inclui recolha de dados com base na internet, assume-se que é uma percentagem positiva e superior ao esperado. Esta não se afastou das taxas alcançadas em estudos semelhantes.

A representatividade da amostra não foi demonstrada 
através da comparação de participantes e não participantes, por não ter sido disponibilizada informação oficial relativa às características do universo dos internos no momento do estudo. Apenas a distribuição por sexo nos internos da região de Lisboa e Vale do Tejo foi partilhada. A única análise binomial possível, baseada nesta informação, mostra uma diferença na distribuição por sexo, com menor proporção de internos homens na amostra. Acresce a aparente infra representação de alguns subgrupos regionais. Assim, presume-se que a amostra não seja representativa, impedindo a generalização dos resultados para toda a população de internos da região sul. Este é um fator limitativo major do estudo.

O Inventário Depressivo de Beck II, como a maioria dos instrumentos de avaliação de sintomas depressivos, apresenta limitações intrínsecas e não permite diagnóstico automático, embora seja um indicador padronizado. Os estudos baseados na resposta a este tipo de questionários estão sujeitos a vieses de memória. Em última instância, a própria presença de depressão pode interferir na vontade e/ou capacidade de responder. Na verdade, alguns autores defendem que a presença de depressão inibe a participação e, portanto, sugerem que a maioria dos estudos com este tipo de desenho metodológico apresentam prevalências subestimadas.

Algumas ocorrências particulares do estudo devem igualmente ser consideradas na interpretação dos resultados. Destaca-se a sazonalidade do período de recolha de dados, coincidente com o período de férias, que pode ter influenciado a taxa e qualidade de resposta. O cronograma inicial precavia esta ocorrência; contudo, algumas disposições externas necessárias ao envio do questionário forçaram o adiamento da data de envio. Semelhante influência poderá associar-se ao pedido do número de identificação no questionário, inibindo a participação apesar de garantida a confidencialidade. Como aspetos de influência positiva destaca-se o cumprimento escrupuloso dos protocolos de confidencialidade e retorno de resultados aos internos.

As limitações atrás designadas, quase todas decorrentes da metodologia escolhida, que aceita uma amostra obtida por autosseleção de participantes, foram previstas e discutidas na fase protocolar. Na literatura, em muitos casos, este assunto tem sido explorado recorrendo a metodologias semelhantes. Este desenho metodológico permite chegar a uma população dispersa por uma vasta área geográfica com recursos ma- teriais e humanos mínimos, sendo este ponto crucial para a exequibilidade do estudo. Deste modo, a escolha metodológica tornou-se aceitável numa perspetiva «piloto», como primeira abordagem de um tema intangível na realidade científica nacional.

Em conclusão, o fator de principal impacto deste trabalho relaciona-se com um maior conhecimento sobre a saúde mental dos internos de MGF em Portugal. Este apresenta uma prevalência de depressão próxima de $19 \%$ numa amostra de 216 internos e sinaliza queixas de cansaço ou fadiga, perda de energia, alterações no padrão de sono e dificuldade de concentração em mais de metade dos respondedores. Tudo isto integrado obriga a relembrar que o médico é também responsável pela saúde do próprio e dos pares, que em última instância se refletirá na saúde da comunidade.

Nesta área de investigação, o futuro passa por evoluir no desenho metodológico dos trabalhos e aprofundar o estudo de fatores predisponentes, fatores protetores, estratégias de coping e de intervenção. 


\section{AGRADECIMENTOS}

A autora agradece à colaboradora externa, Ana Marta Cerol Machado.

\section{REFERÊNCIAS BIBLIOGRÁFICAS}

1. Dyrbye LN, West CP, Satele D, Boone S, Tan L, Sloan J, et al. Burnout among U.S. medical students, residents, and early career physicians relative to the general U.S. population. Acad Med. 2014;89(3):443-51.

2. Sen S, Kranzler HR, Krystal JH, Speller H, Chan G, Gelernter J, et al. A prospective cohort study investigating factors associated with depression during medical internship. Arch Gen Psychiatry. 2010;67(6):557-65.

3. Goebert D, Thompson D, Takeshita J, Beach C, Bryson P, Ephgrave K, et al. Depressive symptoms in medical students and residents: a multischool study.Acad Med. 2009;84(2):236-41.

4. West CP, Huschka MM, Novotny PJ, Sloan JA, Kolars JC, Habermann TM, et al. Association of perceived medical errors with resident distress and empathy: a prospective longitudinal study. JAMA. 2006;296(9):1071-8.

5. Hasanovic M, Herenda S. Post traumatic stress disorder, depression and anxiety among family medicine residents after 1992-95 war in Bosnia and Herzegovina. Psychiatr Danub. 2008 Sep;20(3):277-85.

6. Yi MS, Luckhaupt SE, Mrus JM, Mueller CV, Peterman AH, Puchalski CM, et al. Religion, spirituality, and depressive symptoms in primary care house officers. Ambul Pediatr. 2006;6(2):84-90.

7. Earle L, Kelly L. Coping strategies, depression, and anxiety among Ontario family medicine residents. Can Fam Physician. 2005;51:242-3.

8. Costa AJ, Labuda Schrop S, McCord G, Ritter C. Depression in family medicine faculty. Fam Med. 2005;37(4):271-5.

9. Oriel K, Plane MB, Mundt M. Family medicine residents and the impostor phenomenon. Fam Med. 2004;36(4):248-52.

10. Michels PJ, Probst JC, Godenick MT, Palesch Y. Anxiety and anger among family practice residents: a South Carolina family practice research consortium study. Acad Med. 2003;78(1):69-79.
11. Hainer BL, Palesch Y. Symptoms of depression in residents: a South Carolina Family Practice Research Consortium study. Acad Med. 1998;73(12):1305-10.

12. Godenick MT, Musham C, Palesch Y, Hainer BL, Michels PJ. Physical and psychological health of family practice residents. Fam Med. 1995;27(10):646-51.

13. Reynolds CF 3rd, Clayton PJ. Commentary: Out of the silence: confronting depression in medical students and residents. Acad Med. 2009;84(2):159-60.

14. Caiati ME. Depression and suicide among physicians. News Colorado Phys Health Program. 2006;5(1). Available from: http://www.cphp.org/ documents/depression-suicide.pdf

15. Campos RC, Gonçalves B.Adaptação do Inventário de Depressão de Beck II para a população portuguesa. In: Actas do VIII Congresso Iberoamericano de Avaliação Psicológica, Lisboa, 25 a 27 de julho de 2011.

16. Caldas de Almeida JM, Xavier M. Estudo epidemiológico nacional de saúde mental, $1^{\circ}$ relatório [Internet]. Lisboa: Faculdade de Ciências Médicas da Universidade Nova de Lisboa; 2013. ISBN 9789899857605. Available from: http://www.fcm.unl.pt/main/alldoc/galeria_imagens/Relatorio_Estudo_SaudeMental_2.pdf

\section{CONFLITO DE INTERESSES}

A autora declara não ter conflitos de interesse.

\section{COMISSÃO DE ÉTICA}

Estudo realizado após parecer favorável da Comissão de Ética para a Saúde da ARS de Lisboa e Vale do Tejo.

\section{ENDEREÇO PARA CORRESPONDÊNCIA}

Ana Rita Machado Gomes

R. Comendador F. F. Ribeiro da Costa, $n^{\circ} 111$

2070-597 Vale da Pinta - Cartaxo

Email: rita.ana.gomes@gmail.com

Recebido em 11-01-2015

Aceite para publicação em 05-10-2015

\section{ABSTRACT}

\section{PREVALENCE OF DEPRESSION AMONG FAMILY MEDICINE RESIDENTS IN THE SOUTH OF PORTUGAL}

Objectives: To determine the prevalence of depression and antidepressant use among Family Medicine residents in the Southern Region of Continental Portugal and to study the associations between depression and gender, age, internship year, location of the health center, and type of health care facility.

Study design: Cross-sectional study.

Setting: Family Health Units and Personalized Health Care Units from the south of Portugal.

Participants: Family Medicine residents from the south of Portugal.

Methods: Self-administered questionnaires were sent electronically to Family Medicine residents from the Southern Region of Portugal. The Beck Depression Inventory II was used to measure depression. The Mann-Whitney test, Kruskal-Wallis and Spearman correlation were used to evaluate associations of variables, with a significance level of 0,05 and $95 \%$ confidence intervals.

Results: The response rate was $33 \%$, giving a sample of 216 participants. Almost $80 \%$ of the participants were female. The average age was 30 years old. The residents were equally distributed among the four years of postgraduate training. The majority (88,9\%) worked in Family Health Units and $61,1 \%$ worked in the Lisbon area. About $19 \%$ of the residents had some degree of depression: $8,9 \%$ $(4,7-13,0)$ were classified as mild depression, $6,9 \%(3,2-10,6)$ as moderate depression and $2,9 \%(0,4-5,5)$ as severe depression. Seven percent were treated with antidepressants. Levels of depression were not related to age, gender, internship year or type of health unit. Conclusions: The study represents the first effort to quantify depression among Portuguese Family Medicine residents. It can serve as a starting point for further studies about physicians' mental health, leading to interventions to improve their performance.

Keywords: Residency; Family Medicine; Depression. 